\title{
ESTUDO DAS MOTIVAÇÕES À PRÁTICA DA GINÁSTICA RÍTMICA: ÇONTRIBUIÇÕES DA PESQUISA PARA O RENDIMENTO DESPORTIVO
}

\author{
Patrícia Silveira Fontana \\ Universidade Federal do Rio Grande do Sul, Porto Alegre, Rio Grande do Sul, \\ Brasil
}

\section{Marcus Levi Lopes Barbosa}

Universidade Federal do Rio Grande do Sul, Porto Alegre, Rio Grande do Sul, Brasil

\section{Marcos Alencar Abaide Balbinotti}

Université du Québec à Trois-Riviéres, Trois-Riviéres, Canadá

\section{Carlos Adelar Abaide Balbinotti}

Universidade Federal do Rio Grande do Sul, Porto Alegre, Rio Grande do Sul, Brasil

\section{Resumo}

Este estudo teve por objetivo identificar as dimensões motivacionais mais relevantes que colaboram para o rendimento desportivo em atletas de Ginástica Rítmica (GR). Para tanto, utilizou-se uma amostra de 66 atletas do sexo feminino de 13 a 14 anos. Como medida das dimensões motivacionais, utilizou-se o "Inventário de Motivação à Prática Regular de Atividades Físicas e Esportivas" (IMPRAFE-54). Foram feitas análises descritivas e comparativas. Os resultados indicaram que, para as atletas se manterem motivadas, a prática regular da GR deve ser organizada de forma a proporcionar: Prazer, Sociabilidade, Saúde e Competitividade. Como conclusão sugere-se que os profissionais que atuam nesta área desenvolvam estas dimensões a fim de estimular e manter a motivação de suas atletas.

Palavras-chave: Esportes. Ginástica. Motivação.

\section{Introdução}

pedagogia do treino desportivo se constitui numa área de inves-
tigação das ciências do esporte. Os estudos, de modo geral, situ-
am-se em quatro grandes áreas do conhecimento: técnica desportiva,
treinamento físico-desportivo, aspectos estratégico-táticos dos jogos
de oposição e cooperação, e os fatores psicológicos que influenciam a
performance atlética. A motivação, por exemplo, é um desses fatores
que, em diferentes graus, está presente em toda atividade desportiva.
Por isso, é preciso estudá-la com profundidade, principalmente, por- 
que as principais dimensões motivacionais dos atletas: Controle de Estresse, Saúde, Sociabilidade Competitividade, Estética e Prazer, estão presentes no processo educativo de formação do atleta.

Os resultados das pesquisas científicas promovem, além da produção do conhecimento acadêmico, o desempenho do atleta de alto rendimento. Acredita-se que o treinador de posse do conhecimento das principais motivações que levam os atletas a buscar constantemente o melhor desempenho conseguirá organizar um plano de atividades mais condizente com os interesses dos atletas.

Um aspecto central da teoria do treinamento desportivo, de acordo com Bompa (2002), está no respeito às características específicas de uma modalidade desportiva em particular. A Ginástica Rítmica (GR), por exemplo, caracteriza-se por ser essencialmente feminina. Trata-se de uma atividade competitiva realizada com acompanhamento musical, na qual as atletas executam sua coreografia com elementos corporais unidos aos aparelhos: corda, arco, bola, maças e fita.

Para adquirir performance na GR é preciso muito empenho e dedicação, pois as competições exigem elementos corporais de difícil execução. Por isso, é importante estabelecer um plano de atividades o qual as atletas se mantenham motivadas por um longo período de tempo. A motivação, quando de natureza intrínseca, pode melhorar o interesse pela atividade esportiva, em razão de estar associada à autodeterminação. O comportamento autodeterminado parece estar associado à persistência, ao bem-estar e ao prazer, facilitando a assimilação e a aprendizagem (DECI; RYAN, 1985; 2000; 2002).

Neste artigo, pretende-se identificar as dimensões motivacionais mais relevantes que colaboram para o rendimento desportivo em atletas de GR. Para responder ao objetivo desta pesquisa foi necessário responder duas questões norteadoras: (1) Quais são as dimensões motivacionais que mais motivam as atletas avaliadas?; (2) Há diferenças significativas entre as médias das dimensões motivacionais? Para interpretar os resultados deste estudo sobre motivação, utilizamos como fundamentação teórica, a Teoria da Autodeterminação (DECI; RYAN; 2000; 2002).

\section{Teoria da Autodeterminação: pressupostos teóricos}

A motivação é um fator determinante na preparação desportiva, 
contribuindo para o desempenho e rendimento e auxiliando na permanência e interesse do indivíduo pela atividade.

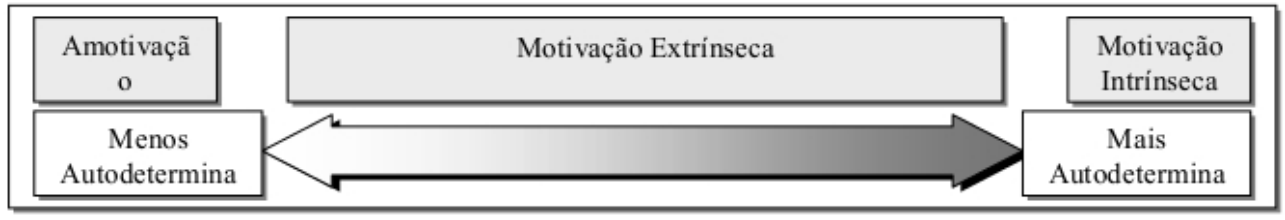

Figura 1: Continuum da Autodeterminação.

A teoria da Autodeterminação (TAD) entende a motivação como um continuum que se estende da amotivação à motivação intrínseca (ver Figura 1). Este contínuo motivacional contempla diferentes níveis de motivação (intrínseca ou extrinsecamente), ou ainda, a amotivação durante uma ação comportamental e/ou prática de uma atividade. Um indivíduo intrinsecamente motivado é aquele que ingressa em uma atividade por vontade própria, diga-se, pelo prazer e pela satisfação de conhecer uma nova atividade (DECI; RYAN, 1985; RYAN; DECI, 2000). Comportamentos intrinsecamente motivados estão associados ao bem-estar psicológico, ao interesse, à alegria e à disposição à tarefa.

Um indivíduo extrinsecamente motivado é aquele que ingressa em uma atividade por expectativas de resultados favoráveis ou outras contingências não necessariamente inerentes à própria atividade (RYAN; DECI, 2000). Comportamentos extrinsecamente motivados podem ter grande variação em relação ao grau de autonomia do sujeito e de sua capacidade de tratamento da informação recebida. Para uma compreensão global da motivação extrínseca, pode-se dizer que ela compreende quatro categorias distintas, mas relacionáveis: Regulação Externa, Regulação Introjetada, Regulação Identificada, e Regulação Integrada.

Além das motivações de natureza intrínseca e extrínseca, a TAD entende que a amotivação também afeta as ações comportamentais dos indivíduos. A amotivação é um estado motivacional que pode ser encontrado em indivíduos que não estão suficientemente aptos a identificar bons motivos para realizar uma "determinada" atividade, ou seja, não está disposto a agir naquela direção, a realizar aquela ação, a atingir aquele alvo, a alcançar aquele objetivo (RYAN; DECI, 2000). Pode-se dizer que para alguns indivíduos, em seus próprios pontos de vista, algumas atividades não trazem o benefício desejado (esperado, 
determinado, identificado), ou eles não se sentem aptos a realizá-las de modo satisfatório, ou qualquer outro motivo que os faz negar a ação da obtenção do prazer (dimensão representativa do comportamento autônomo e autodeterminado) gerado pela atividade realizada.

Uma vez expostos os diferentes níveis de determinação do comportamento, é importante que se diga que o estado extrinsecamente motivado não corresponde a um comportamento necessariamente negativo. De acordo com Ryan et al. (1997), os motivos extrínsecos podem possuir graus maiores ou menores de autonomia e de liberdade cognitivo-intelectual. Quanto maior esse grau, mais perto da autodeterminação o indivíduo se encontra. Quanto menor o grau de autonomia e de liberdade cognitivo-intelectual, maior será a distância que o indivíduo se encontra da amotivação. Por um lado, estima-se que a amotivação seja um dos importantes fatores que contribuem para que o atleta abandone, prematuramente, sua carreira no esporte; por outro lado, e considerando que motivos intrínsecos possuem caráter fundamentalmente autodeterminável, estima-se que são esses atletas que obterão grande probabilidade de sucesso em suas carreiras.

Assim, conhecidas as implicações e conseqüências do processo motivacional, justifica-se que os motivos à prática de atividades esportivas sejam conhecidos e estudados. De forma geral, os diversos modelos de avaliação dos motivos são divergentes. Enquanto alguns modelos propõem ampla variedade de dimensões, como no caso de Markland e Ingledew (1997), que propõe um modelo em 14 dimensões, outros propõem dimensões menos numerosas, como no caso de Fernández e Solá (2001), que indica que a motivação poderia ser suficientemente explicada por um modelo de três dimensões.

Uma recente revisão destes modelos indicou que seis dimensões parecem ser suficientes para explicar os motivos à prática de atividades físicas e esportivas. Trata-se do modelo proposto por Balbinotti e Barbosa (2006), que inclui as seguintes dimensões motivacionais: Controle de Estresse, Saúde, Sociabilidade, Competitividade, Estética e Prazer. O modelo foi testado em diferentes grupos de praticantes de atividades físicas e esportivas de ambos os sexos e em diferentes idades (FONTANA, 2008; JUCHEM, 2006; SALDANHA, 2008). O objetivo central desses estudos foi verificar, entre as dimensões citadas, aquelas que mais motivam os praticantes. Acredita-se que a partir dos resultados obtidos será possível melhorar consideravelmente a proposta pedagógica orientada para o treinamento. A seguir serão apre- 
sentados a metodologia, os resultados e as interpretações subsequentes ao estudo realizado com atletas da GR.

\section{Material e métodos}

Caracterização do Estudo

Trata-se de um estudo descritivo-exploratório, que pretende identificar as principais dimensões motivacionais que levam à prática regular da GR que podem contribuir para o rendimento das atletas na modalidade.

População e Amostra

O estudo contou com a participação de uma amostra de 66 atletas juvenis de GR do sexo feminino de 13 e 14 anos do Rio Grande do Sul, participantes de Campeonatos Brasileiros e Campeonatos Estaduais, filiadas à Federação Riograndense de Ginástica e à Confederação Brasileira de Ginástica. Os critérios de seleção da amostra foram os da disponibilidade e da acessibilidade (MAGUIRE; ROGERS, 1989).

Instrumentos

Para este estudo foram utilizados dois instrumentos. Para o controle das variáveis, idade e tempo de prática se utilizou o "Questionário de Identificação das Variáveis de Controle" (QIVC) que está incluso no cabeçalho do Inventário de Motivação à Prática Regular de Atividades Físicas e Esportivas. Como medida das dimensões motivacionais para a prática regular de atividades físicas e esportivas, utilizou-se o "Inventario de Motivação à Prática Regular de Atividades Físicas e Esportivas" (IMPRAFE-54), (BALBINOTTI; BARBOSA, 2006). O IMPRAFE-54 é composto por 54 itens agrupados 6 a 6 , seguindo a sequência das dimensões a serem estudadas (Controle de Estresse, Saúde, Sociabilidade, Competitividade, Estética e Prazer). As respostas aos itens do IMPRAFE-54 seguem uma escala bidirecional do tipo Likert, graduada em 5 pontos, indo de "isto me motiva pouquíssimo" (1) a "isto me motiva muitíssimo" (5). As propriedades métricas deste instrumento foram exploradas em um extenso estudo 
com 1377 sujeitos (BARBOSA, 2006). Foram avaliadas a validade fatorial confirmatória $(\chi 2 / \mathrm{gl}=2,31 ; \mathrm{GFI}=0,952 ; \mathrm{AGFI}=0,948 ; \mathrm{RMS}$ $=0,058)$ e a consistência interna (índices Alphas de Cronbach variando de 0,82 a 0,94$)$ com resultados bastante satisfatórios. Este instrumento tem sido utilizado em diferentes grupos de praticantes de atividades físicas e esportivas de ambos os sexos e em diferentes idades (CAPOZZOLI, 2006; FONTANA, 2008; JUCHEM, 2006; SALDANHA, 2008).

\section{Procedimentos}

Inicialmente, o projeto de pesquisa foi submetido à análise do Comitê de Ética e Pesquisa da Universidade Federal do Rio Grande do Sul (UFRGS) e aprovado na reunião no56, ata $\mathrm{n}^{\circ} 136$ de 24 de setembro de 2009, sob o número 2008133.

Após sua aprovação, para a realização da coleta foi necessário o Termo de Concordância das Instituições (TCI), para autorizar efetuar as coletas; o Termo de Consentimento Livre e Esclarecido (TCLE) para os responsáveis legais pelas atletas e o contato com os treinadores e os pais destas atletas. A aplicação do IMPRAFE-54 ocorreu entre os meses de novembro de 2009 a fevereiro de 2010. Os inventários foram respondidos individualmente e aplicados coletivamente a todas as praticantes de GR que se disponibilizaram a participar do estudo, no local de treinamento, após a aula. A aplicação foi feita em um único momento, após dez minutos do término da preparação física das atletas. Os inventários foram aplicados pela própria pesquisadora. Entre as instruções de preenchimento e a aplicação dos inventários levou-se em torno de trinta minutos. A pesquisadora acompanhou as participantes em todo o processo de preenchimento dos inventários, esclarecendo dúvidas que pudessem surgir no decorrer deste processo. Os critérios de seleção que foram utilizados na coleta foram: a atleta deveria ser filiada à Federação Riograndense de Ginástica e à Confederação Brasileira de Ginástica e ser participante dos Campeonatos Estaduais e Brasileiros nas categorias de 13 e 14 anos. Conforme acordado com as atletas, os nomes das participantes que responderam o IMPRAFE-54 não serão revelados.

Quanto aos procedimentos estatísticos, o banco de dados foi construído no pacote estatístico SPSS 15.0. Foram feitas as análises descritivas e comparativas dos dados. Primeiramente foi calculado a 
média (estatística de tendência central), a normalidade (K-S), a assimetria, o achatamento (estatística de distribuição) e o desvio padrão, os valores mínimos e máximos (estatística de dispersão). Por fim, foram comparadas as médias das dimensões motivacionais por meio do Teste t pareado com o propósito de verificar se havia diferenças significativas entre as médias.

\section{Apresentação e discussão dos resultados}

A fim de atender ao objetivo deste estudo, procedeu-se à exploração dos escores obtidos através do IMPRAFE-54, segundo princípios norteadores comumente aceitos na literatura especializada (PESTANA; GAGEIRO, 2003; REIS, 2001). Após, foram apresentados os resultados, sucessiva e sistematicamente.

Uma análise inicial, considerando os valores nominais das médias, indicou que as dimensões motivacionais ficaram ordenadas da seguinte forma: Prazer, Sociabilidade, Saúde, Competitividade, Estética e Controle de Estresse (ver Figura 2).

Análises descritivas mais detalhadas (ver Tabela 1) indicam que as médias variaram consideravelmente, indo de 16,11 (na dimensão Controle de Estresse) a 32,19 (na dimensão Prazer). Isso indica forte variação na intensidade das dimensões motivacionais.

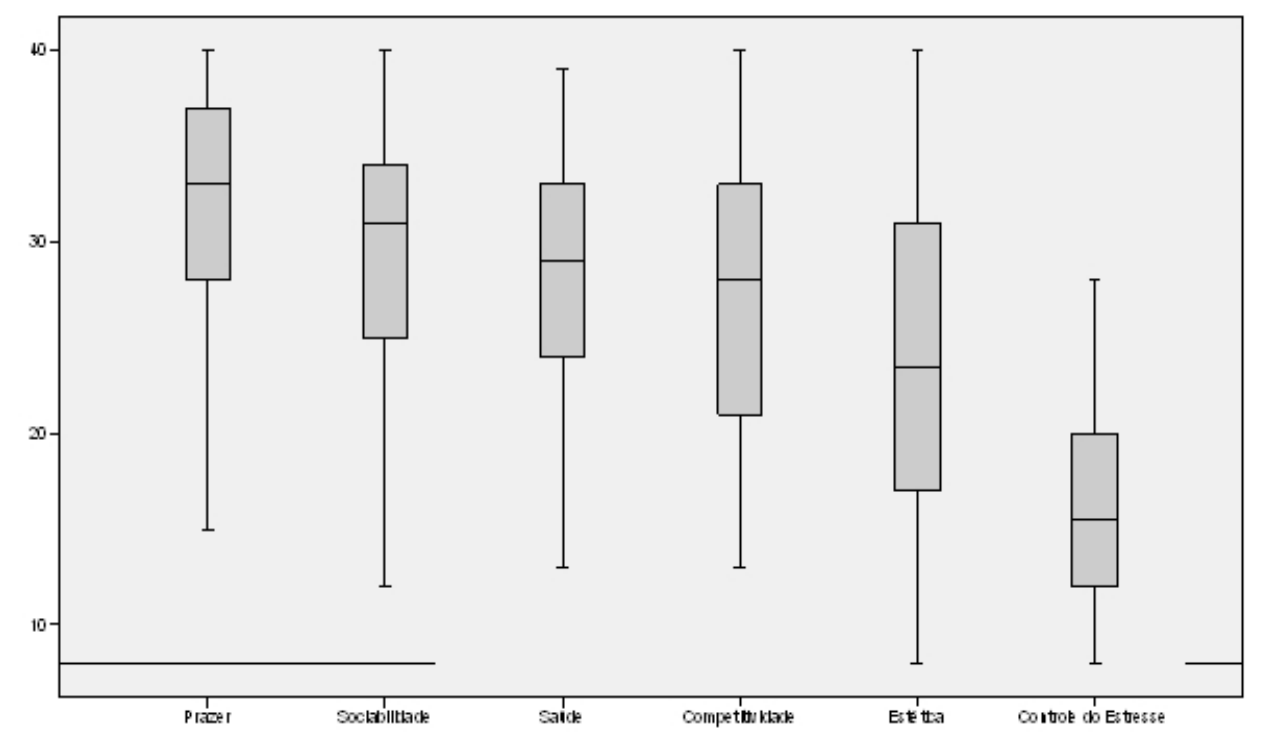

Figura 2: Distribuições das dimensões motivacionais à prática da GR. 
Quanto aos valores extremos, esperava-se que variassem de 8 a 40 . O que se observou é que os valores mínimos afastaram-se do esperado nas seguintes dimensões: Prazer (15), Saúde (13), Competitividade (13) e Sociabilidade (12), indicando que nenhum sujeito nesta amostra encontra-se amotivado quando se trata destas quatro dimensões motivacionais. No que diz respeito aos valores máximos, apenas na dimensão Controle do Estresse (28) os valores afastaram-se do esperado, indicando que nenhum sujeito desta amostra é motivado por esta dimensão (visto o grande afastamento em questão). Uma análise da distribuição indica distribuições simétricas, mesocúrticas e com aderência à normalidade em todas as dimensões, exceto na dimensão Prazer. Neste caso, os altos níveis de motivação provocam uma assimetria na curva, suficientemente forte para provocar sua não adesão à normalidade. O mesmo tipo de distorção (decorrente dos elevados índices de motivação) pode ser observado na dimensão Sociabilidade, neste caso, não tão forte, de maneira que adesão à normalidade não é afetada.

Tabela 1: Análises descritivas das seis dimensões motivacionais avaliadas.

\begin{tabular}{lllllll}
\hline \multirow{2}{*}{ Dimensão } & $\begin{array}{l}\text { Tendência } \\
\text { Central } \\
\text { (dispersão) }\end{array}$ & $\begin{array}{l}\text { Tendência } \\
\text { Não-central }\end{array}$ & Normalidade & Assimetria & Achatamento \\
\cline { 2 - 7 } & Média (DP) & Mínimo & Máximo & K-S (sig.) & Skewness/Erro & Kurtosis/Erro \\
\hline Prazer & $32,19(5,73)$ & 15,00 & 40,00 & $0,13(0,009)$ & $-1,76$ & $-0,29$ \\
Sociabilidade & $29,53(7,04)$ & 12,00 & 40,00 & $0,09\left(0,200^{*}\right)$ & $-1,94$ & 0,29 \\
Saúde & $28,38(6,26)$ & 13,00 & 39,00 & $0,06\left(0,200^{*}\right)$ & $-0,91$ & $-0,64$ \\
Competitividade & $27,22(7,06)$ & 13,00 & 40,00 & $0,08\left(0,200^{*}\right)$ & $-0,13$ & $-1,50$ \\
Estética & $23,72(9,47)$ & 8,00 & 40,00 & $0,10\left(0,200^{*}\right)$ & $-0,16$ & $-1,76$ \\
Controle Estresse & $16,11(5,44)$ & 8,00 & 28,00 & $0,10(0,086)$ & 1,30 & $-1,05$ \\
\hline
\end{tabular}

Obs: * valor máximo para significância desta estatística.

Com o objetivo de verificar a presença de diferenças significativas entre as médias das dimensões e descrever o perfil motivacional das atletas, utilizou-se um teste $\mathrm{t}$ pareado. $\mathrm{O}$ teste $\mathrm{t}$ foi escolhido porque a homogeneidade das variâncias do grupo total das ginastas foi rejeitada pelo teste de Mauchly's $(\mathrm{p}<0,01)$. Os resultados indicam que a dimensão Prazer motivou as ginastas de forma mais significativa ( $\mathrm{p}<$ $0,05)$ que as demais dimensões, sendo a dimensão que melhor representa o perfil motivacional das atletas avaliadas. Seguindo-se a esta, um grupo de três dimensões indissociáveis estatisticamente $(\mathrm{p}>0,05)$, formados pelas dimensões Sociabilidade, Saúde e Competitividade. As duas dimensões seguintes (Estética e Controle de Estresse) apresentam diferença significativa $(p>0,05)$ deste grupo e entre si. 
Esta informação tem implicações importantes no planejamento das atividades de treino, pois quem as planeja está interessado em fazê-lo de modo a manter as atletas motivadas. Como se vê o Prazer em fazer a atividade desportiva em questão é o que mais motiva estas atletas. Praticar a atividade lhes proporciona Prazer, e o Prazer é o protótipo da motivação intrínseca. Ele está associado ao bem-estar, à persistência, à autoestima e à disposição à tarefa.

Uma das características positivas deste esporte é que em todas as sessões de treinamento se pratica a modalidade. Como se demonstrou anteriormente, que é fonte de Prazer para as suas praticantes. Mesmo que em alguns momentos como a preparação física específica realizada através do ballet e o treinamento de força, não sejam tão prazerosos, é importante destinar um período de treino para a prática do esporte.

Ainda assim, sabe-se que este esporte exige movimentos artísticos, coordenação motora complexa e alto grau de flexibilidade, de forma que as longas seções de flexibilidade e o forte trabalho muscular, característicos deste esporte, por motivos óbvios, não podem ser negligenciados. $\mathrm{O}$ resultado deste estudo indica um grupo de três dimensões motivacionais (Sociabilidade, Saúde e Competitividade) que se segue ao Prazer e nos fornece indicativos preciosos sobre como manter a motivação, mesmo fora do período de prática do esporte propriamente dito.

Quanto à Sociabilidade, primeira das três dimensões motivacionais deste grupo, trata-se da atenção que o treinador deve dar ao planejamento destes períodos, de maneira a promover atividades no aquecimento e na preparação física onde a socialização seja fomentada. É preciso planejar atividades em grupo, atividades colaborativas, propiciar a interação social, criar um ambiente de parceria e cumplicidade entre as atletas. Atividades como estas têm importante papel na manutenção da motivação elevada.

Quanto à Saúde, segunda dimensão motivacional deste grupo, sugere-se que o treinador possa melhor orientar sua atleta sobre a importância da realização dos diferentes tipos de treinamentos e que benefícios estes podem proporcionar a sua saúde. Em um trabalho multilateral, pode não ficar muito claro para a atleta o porquê de realizar determinadas atividades que não parecem estar diretamente relacionadas à GR. Isso poderia ser encarado como uma perda de tempo (e ser desmotivador). As razões e objetivos podem estar claras "na ca- 
beça" do treinador, mas precisam estar claras também para as atletas (já que Saúde é uma dimensão motivacional importante para elas).

Quanto à Competitividade, poder-se-ia pensar que seria alimentada unicamente através das participações em competições. Isso é um fator importante de incremento desta motivação (afinal de contas é para competir que se treina). Ainda assim, durante as atividades de treinamento a competição pode estar presente. É importante que o treinador promova atividades competitivas nos mais diferentes momentos do treino (com "brincadeiras" e jogos colaborativos ou não), desenvolvendo a Competitividade e utilizando-a como ferramenta de caráter educativo. A competição é uma importante fonte de feedback e tem relevante papel na percepção de competência de atletas. Trata-se de uma característica importante e inerente a atividade esportiva que não deve ser subestimada nem superestimada.

Quanto à Estética e o Controle de Estresse, tratam-se de dimensões motivacionais que, de acordo com os resultados, não desempenham papel importante na motivação destas atletas. No caso da Estética, se poderia pensar que é uma condição para prática desta modalidade desportiva e não uma dimensão motivacional para praticá-la. No caso do Controle de Estresse, aspectos relacionados à fase do desenvolvimento poderiam explicar a pouca importância desta dimensão motivacional à prática do esporte nestas adolescentes.

Estes resultados podem ser particularmente importantes para os profissionais que trabalham diretamente na preparação de atletas de GR. Destaca-se que estes profissionais deveriam considerar as diferenças estatisticamente significativas aqui apresentadas e criar modelos de orientação à prática da GR.

\section{Conclusões}

A prática pedagógica estabelecida no âmbito do treino desportivo precisa ir além dos conhecimentos advindos da prática cotidiana. Durante décadas, coube ao treinador, na maior parte dos casos um exatleta, a responsabilidade de orientar o atleta, que almejava o alto rendimento desportivo. Não se quer aqui desvalorizar o conhecimento adquirido por esses profissionais ao longo de uma história de vida dedicada ao esporte; pelo contrário, acredita-se que, os conhecimentos adquiridos pelo ex-atleta ao longo de sua carreira enquanto atleta são legítimos e inquestionáveis. No entanto, podem ser insuficientes, ten- 
do em vista que para atingir a performance de alto nível desportiva na atualidade é preciso buscar conhecimentos para além das vivências práticas. Nesse contexto, os resultados obtidos nas pesquisas científicas podem contribuir de forma relevante para a aquisição de índices elevados de rendimento do atleta.

Através dos resultados dessa pesquisa que trata da motivação em jovens praticantes de GR, espera-se contribuir com o trabalho desenvolvido pelos treinadores, dirigentes, psicólogos, entre outros que atuam no contexto esportivo. O período necessário para a formação de uma atleta de GR de alto nível tem duração média de 8 a 10 anos. Muitas perdem a motivação durante essa trajetória caracterizada por esforços ininterruptos de ordem física e psicológica. De alguma forma, é preciso mantê-las motivadas, mesmo com inúmeras derrotas que, sem exceção, marcam a carreira desportiva dessas jovens. Com os resultados obtidos neste estudo foi possível identificar que o Prazer, a Sociabilidade, a Saúde e a Competitividade são as dimensões motivacionais que mais motivam as atletas para a prática regular da GR. Assim, sugere-se que os profissionais que atuam nesta área desenvolvam em seu planejamento de treino estas dimensões motivacionais aqui relacionadas com o propósito de estimular e manter a motivação de suas atletas.

Study of the motivations for the practice of rhythmic gymnastics: research contributions to performance sport

\begin{abstract}
This study aimed to identify the most relevant motivational dimensions that contribute to athletic performance in athletes of Rhythmic Gymnastics (RG). For this purpose, we used a sample of 66 female athletes from 13 to 14 years. To measure the motivational dimensions, we used the "Motivational Inventory to Regular Physical Activity and Sports" (IMPRAFE-54). Were conducted descriptive and comparative analysis. The results indicated that for athletes to stay motivated, the regular practice of RG should be organized so as to provide: Pleasure, Sociability, Health and Competitiveness. In conclusion it is suggested that professionals working in the field to develop these dimensions to stimulate and maintain the motivation of their athletes.
\end{abstract}

Keywords: Sports. Gymnastics. Motivation. 
Estudio de la motivación para práctica de la gimnasia rítmica: contribuciones de la investigación para el rendimiento deportivo

\section{Resumen}

Este estudio tuvo como objetivo identificar las dimensiones de motivación más importantes que contribuyen para el rendimiento deportivo en atletas de gimnasia rítmica (GR). Para ello, se utilizó una muestra de 66 atletas femeninas de 13 a 14 años. Como las dimensiones de motivación, se utilizó el "Inventario de Motivación de la Actividad Física y Deportiva" (IMPRAFE-54). Se realizaron análisis descriptivo y comparativo. Los resultados indicaron que los atletas a mantener la motivación, la práctica regular da GR debe organizarse a fin de proporcionar: el Placer, la Sociabilidad, la Salud y la Competitividad. En conclusión, se sugiere que los profesionales que trabajan en el campo para el desarrollo de estas dimensiones con el fin de estimular y mantener la motivación de sus atletas.

Palabras clave: Deportes. Gimnasia. Motivación.

\section{Referências}

BALBINOTTI, M. A. A.; BARBOSA, M. L. L. Inventário de motivação à prática regular de atividade física (IMPRAFE-54). Laboratório de Psicologia do Esporte - Escola Superior de Educação Física, Universidade Federal do Rio Grande do Sul, Porto Alegre, 2006.

BARBOSA, M. L. L. Propriedades métricas do Inventário de Motivação para a Prática Regular de Atividade Física (IMPRAF-126). 2006. 140 f. Dissertação (Mestrado em Ciências do Movimento Humano) - Escola Superior de Educação Física, Universidade Federal do Rio Grande do Sul, Porto Alegre, 2006.

BOMPA, T. O. Periodização: Teoria e metodologia do treinamento. São Paulo: Phorte, 2002.

CAPOZZOLI, C. J. Motivação à prática regular de atividades físicas: Um estudo com praticantes em academias de ginástica em Porto Alegre. 2006. 153 f. Dissertação (Mestrado em Ciências do Movimento Humano)-Escola Superior de Educação Física, Universidade Federal do Rio Grande do Sul, Porto Alegre, 2006.

DECI, E. L.; RYAN, R. M. Intrinsic motivation and self-determination in human behavior. New York: Plenum, 1985. 
. The "what" and "why" of goal pursuits: human needs and the self-determination of behavior. Psychological Inquiry, v. 11, n. 4, p. 227-268, 2000.

. The paradox of achievement: The harder you push, the worse it gets. In: ARONSON, J. (Ed). Improving academic achievement: contributions of social psychology. New York: Academic Press, 2002. p. 59-85.

FERNÁNDEZ, I. C.; SOLÁ, I. B. Dimensiones de los motivos de práctica deportiva de los adolescentes valencianos escolarizados. Apunts, v.63, p. 22-29, 2001.

FONTANA, P. S. et al. Motivação à prática regular de atividades físicas: um estudo exploratório com atletas juvenis de ginástica rítmica (13 a 14 anos). In: CONGRESSO DO DESPORTO E EDUCAÇÃO FÍSICA DOS PAÍSES DE LÍNGUA PORTUGUESA, XII, 2008, Porto Alegre. Anais.... Porto Alegre: UFRGS, 2008. 1 CD- ROM.

JUCHEM, L. A Motivação à prática regular de atividades físicas: um estudo sobre tenistas brasileiros infanto-juvenis. 2006. $111 \mathrm{f}$. Dissertação (Mestrado em Educação Física)-Curso de Educação física, Departamento de Educação Física, UFRGS, Porto Alegre, 2006

MAGUIRRE, T. O.; ROGERS, W. T. Proposed solutions for non randomness in educational research. Canadian Journal of Education, v. 14, n. 2, p. 170-181, 1989.

MARKLAND, D.; INGLEDEW, D. K. The measurement of exercise motives: factorial validity and invariance across gender of a revised exercise motivations inventory. British Journal of Health Psychology, v. 2, p. 361-376, 1997.

PESTANA, M. H.; GAGEIRO, J. G. Análise de dados para ciências sociais: a complementaridade do SPSS. 3. ed. Lisboa: Silabo, 2003.

REIS, E. Estatística multivariada aplicada. 2. ed. Lisboa: Silabo, 2001.

RYAN, R. M.; DECI, E. L. Intrinsic and extrinsic motivations: Classic definitions and new directions. Contemporary Educational Psychology, v. 25, n. 1, p. 54-67, 2000. 
RYAN, R. M. et al. Intrinsic motivation and exercise adherence. International Journal of Sport Psychology, Roma, v. 28, p. 335-354, 1997.

SALDANHA, R. P. Motivação à prática regular de atividades físicas: um estudo com atletas de basquetebol infanto-juvenis (13 a 16 anos). 2008. 119 f. Dissertação (Mestrado em Ciências do Movimento Humano)-Escola Superior de Educação Física, Universidade Federal do Rio Grande do Sul, Porto Alegre, 2008.

Recebido em: $14 / 01 / 2012$

Revisado em: 28/04/2012

Aprovado em: 05/10/2012

Endereço para correspondência

cbalbinotti@terra.com.br

Carlos Adelar Abaide Balbinotti

Universidade Federal do Rio Grande do Sul

Escola Superior de Educação Física.

Rua Felizardo, 750

Jardim Botânico

90690-200 - Porto Alegre, RS - Brasil 